\title{
Using on-line and CD-ROM database archives as an adjunct to teaching survey research methods
}

\author{
DOUGLAS B. EAMON \\ University of Wisconsin, Whitewater, Wisconsin
}

\begin{abstract}
Many undergraduate courses in research methods include a section on surveys. A number of practical issues can interfere with the design and implementation of student survey research and often preclude the completion of well-designed survey studies. This paper examines the use of on-line and CDROM databases as an alternative to student-designed survey instruments. Problems faced by student survey researchers can often be addressed by the use of such databases, rather than by "live" surveys. Data from experimental research collected on line are also available and downloadable for analysis, providing even greater flexibility for analysis. Advantages and problems of using archival databases in the teaching of survey research are considered. A brief list of databases that may be suitable for student research is provided, and a list of URLs, including where and how to obtain archives, is given.
\end{abstract}

An important component of many undergraduate courses in research methods is a section on surveys. Textbooks commonly include one or more chapters on surveys that discuss problem identification, question format and instrument design, methods of administration, sampling procedures, and analysis and reporting of results (e.g., Heiman, 1995; Ray, 2000; Vadum \& Rankin, 1998). Students enrolled in these courses presumably may be expected to apply this information in an associated laboratory or in another undergraduate setting by constructing a survey, administrating it, and analyzing its results. However, survey research that enables students to pursue their interests is often impeded by practical difficulties that interfere with and often preclude the conduct of adequate projects. Especially if students design their projects from the ground up, at each step in the process they are likely to encounter pitfalls that prevent the completion of well-designed survey studies.

\section{A Survey of Student Surveys: Problems and a Possible Solution}

Students' interests often include problems, such as sexual behavior or drug and alcohol use, that necessitate a more than cursory review (a "Full Board Review") by an Institutional Review Board (IRB), resulting in prohibitively long delays or even rejection of their proposals. Obtaining a representative random sample of a target population can especially raise problems, particularly if the student wishes to generalize to a population of substantial size (e.g., college students, young men/women, etc.). These problems are compounded when the study must be completed within one semester and are even fur-

Correspondence concerning this article should be addressed to D. B. Eamon, Department of Psychology, University of Wisconsin, Whitewater, WI 53190 (e-mail: eamond@uwwvax.uww.edu). ther exacerbated when the project is one of several that must be completed within a semester.

Many of the teaching aims of a survey project can be attained when students are able to use data archives available on the World-Wide Web (WWW) or on CD-ROMs, rather than create and execute the entire project themselves. Data contained within these databases are themselves often the product of surveys and so reflect many of the characteristics of survey research. The availability and use of archival data and the advantages and disadvantages of secondary analysis of archival research as an alternative to individually designed surveys provide an opportunity for students to understand important aspects of both survey methods and archival research.

Available archives vary widely in content and structure, ranging from U.S. Census data to small data sets focusing on local or regional concerns. Longitudinal data, such the National Longitudinal Survey of Youth (NLSY; Center for Human Resources Research, 1998) may include 20 or more years of data. Larger data sets commonly provide a means for variable selection, either on line or by using selection tools provided with CD-ROMs, so that only those variables that pertain directly to the student's project can be selected.

Recognizing the potential for data storage and retrieval made possible by the WWW, the Behavior Science Database Consortium (1999) proposed the development of a database for use by researchers and teachers in psychology. Unlike most other databases, this collection will include data collected under controlled experimental conditions, as well as surveys and other methods of data collection. A similar project has been on-going at the University of Essex since 1967 (Oehlmann, 1998). PsychExperiments (Williams, McGraw, \& Tew, 1999) is an online cognitive and social psychology laboratory site from which numerous experiments may be run on line. Browsers of this site can run themselves in experiments and 
download data for analysis. These databases promise to greatly expand archival research opportunities for students and researchers and suggest that database archives will play an increasingly important role in the future training of psychologists, underlining the need for the training of students in archival and database research.

\section{Pedagogy of "Live" Surveys Versus Archival Research}

There are important differences between survey research and archival research, and some learning experiences obtained by using survey research may be made more difficult or unreachable when archival databases are used. For example, students commonly get data "feedback" from respondents who fail to understand the wording of survey questions written by the student. The use of secondary analysis brings additional problems that need to be addressed (see McCall \& Appelbaum, 1991). Nonetheless, my experience has convinced me that, on balance, students obtain more benefit from database survey projects than from independent survey development and data collection "from scratch," and the likelihood of even greater future access to on-line databases provides still further justification for a shift in focus from locally conducted surveys to data archives. The use of the WWW to collect survey and research data (see Hewson, Laurent, \& Vogel, 1996, for a discussion of some methodological issues) provides additional opportunities for the creation of on-line databases that make these data accessible.

Although database archives have been available for some time to researchers, very little information is available on the use of database archives in teaching research methods. Pachnowski, Newman, \& Jurczyk (1997) discuss the use of databases but include only a few of the archives available, omit several of the largest and most comprehensive archives, and provide little information about where to search for additional resources. Drake and Stahl (1995) focus on the hardware required for the development of an instructional laboratory exclusively designed around the use of U.S. census data. Few of the popular textbooks in research methods address archival research much beyond a paragraph or two (e.g., Bordens \& Abbott, 1996; Heiman, 1995; Liebert \& Liebert, 1995; Ray, 2000), and one of the most popular texts does not even mention archival research at all (Elmes, Kantowitz, \& Roediger, 1999). This paper will help fill this apparent gap and remind teachers of the potential archive databases have for the teaching of research methods, particularly as an adjunct or alternative to teaching survey methods.

To this end, I will first consider some of the problems that students face in the development of survey instruments and the collection of survey data and will indicate how the use of archival databases as an alternative can help overcome these problems. Some problems associated with the instructional use of archived databases will also be considered. A brief examination of several data- bases suitable for use in an undergraduate course will be provided, and a list of sources and URLs that will enable the instructor to locate appropriate archives for his/her own use will be given.

The following observations pertain to the use of data archives in teaching research methodology, especially as an adjunct or alternative to student-designed surveys. For more general discussions of methodological issues arising from database research and WWW-based data acquisition, see Hewson et al. (1996), McCall and Appelbaum (1991), and Singleton, Straits, and Straits (1993).

\section{Student Projects: Problems Addressed Using Database Archives}

Problem identification. The most commonly selected topics for survey research in my classes have been, for many years, (1) sexual attitudes and sexual behavior (especially of young people and/or students), (2) drug and alcohol use (especially by young people and/or students), and (3) indicators of possible psychological and emotional disorders by people under stress (especially young people and/or students). Each of these is an important topic and a legitimate research interest with substantial background literature, but students do not engage in research on any of these topics on my campus. The reason is that student-designed surveys that probe other students about these areas do not pass IRB review. In the IRB risk/benefit analysis, the risk of inadvertent release of personal information, misinterpretation or misuse of information by unsophisticated student researchers, psychological distress to the respondents, and, sometimes, possible community "political" fallout from release of even general summaries of findings outweigh the likely benefits, which are often not clearly articulated by the student researcher. Even in research in which none of these risk factors is involved (preference studies of entertainment, courses, leisure time use, etc.), the delay between submission of the materials to the IRB and receipt of permission to proceed makes completion of the projects as part of a one-term course difficult. Longitudinal studies are, of course, out of the question in this context.

However, numerous database archives contain variables related to sexual behavior. Variables include frequency of sexual intercourse and orgasm, age of first intercourse, sexual preferences, and so on. Drug and alcohol use, emotional responses of various sorts, including numerous questions designed to assess aspects of personality and personal development (e.g., self-esteem, internality-externality), as well as reported stress, are available on some special interest databases, and most of these variables are available on a single large database, the NLSY (Center for Human Resources Research, 1998). Using such databases makes it possible for students to collect information on, say, the relationship between age of first sexual intercourse, drug use, and self-esteem. Selection of such topics also provides the instructor with an opportunity to suggest other factors that may contribute 
to the target behavior to be examined and, thereby, focus the student's attention on the complexity of human behavior and the need for careful procedures for analysis.

Question format and instrument design. The wording of questions and the design of the survey instrument can dramatically affect respondents' answers. Introductory level students very often fail to consider the many problems attendant in survey design and produce survey instruments that lack validity, reliability, and credibility. Of course, careful supervision of student projects provides an opportunity for faculty to teach appropriate wording and design and to refer students to good examples. Students can learn much from this experience. At the same time, the survey instruments on which database archives are based are usually prepared by professional researchers, and the data collected are likely to have greater reliability and validity than student-designed instruments, as well as to provide instructive examples of question format and design.

Sampling. Perhaps the most salient feature of past survey research projects conducted by students in my classes is that the population to which they wish to generalize is not sampled appropriately. Whether this population is young people, college students, college students at our university, psychology majors, male or female students at the university, male or female psychology students at the university, or any other reasonably sized population not directly accessible to my students, the difficulties in obtaining anything but a nonprobability sample are nearly insurmountable in the context of a one-term course. A major problem in nearly all cases is obtaining a sufficiently large sample to enable a statistical analysis sufficiently powerful to draw conclusions about the target population. In contrast, several archival databases have thousands of cases representing random samples from a variety of large populations.

Administration. Methods of administration can also pose a problem, depending on the target population and the technique that is deemed the most appropriate. For surveys of enrolled students, telephone surveys may be the easiest and most practical, since they can be conducted from the student's home in a relatively short time. Mailed surveys pose a more difficult problem, because one might expect to wait at least 10 days to 2 weeks for a written survey to be returned. More serious problems may arise when interviews are determined to be the preferred method, because respondents first have to be reached by telephone or mail, an interview date and time arranged, and the interview scheduled and completed. With database archives, however, even longitudinal studies that include data collected over many years may be available.

Related literature. When students select problems, they are often either overwhelmed by the amount of information available or unable to locate sources that connect closely to the ideas they wish to develop. They waste much time casting around in fruitless searches for journal articles that provide instances of what an interesting and researchable problem might be and of how to pro- ceed with its implementation. However, several archives give references to articles, dissertations, reports, and other work based on the archive-for example, the NLSY (Center for Human Resources Research, 1998) and the General Social Survey (GSS; Davis \& Smith, 1997). For some topics, hundreds of such reports are cited, and, like the $N L S Y$, the GSS provides abstracts on line. My students have found these sources invaluable in their search for information on how to analyze the data they collect and to understand and interpret them, as well as in their initial search for ideas for research.

\section{Speed and Ease of Revision of Data Collected}

In every case, the use of database archives as a data source greatly enhances the ease and speed with which data can be collected. Should a student find that he/she has forgotten to obtain data that were present in the data set and that may have enhanced the interpretation of the results, it is a simple matter to return to the archive, add the additional variables, and perform the data extraction again. It is not unusual for students to perform numerous extractions in the process of determining the variables that provide a satisfactory explanation and interpretation of the phenomenon the student wishes to explore.

\section{Special Problems in Using \\ Archival Data in Teaching}

Availability of information related to student interests. Although use of archival data may help overcome some problems students face in the preparation of projects, archives also present special problems that need to be addressed. First and most obvious, because they are not written by the student researcher, the questions may not tap precisely the knowledge that the student seeks to uncover or may take a form that presents difficulty for data analysis or interpretation.

Data and database accessibility. Another problem is the accessibility of the data. Some databases-for example, many of those available at the National Center for Education Statistics - require documentation of the researcher's status as a bona fide researcher. The GSS on-line database (Davis \& Smith, 1997) is sometimes not accessible using the WWW, presumably owing to server overloads; this is especially disruptive - and likely - if the instructor asks all of the students enrolled to attempt to access the database simultaneously from the same laboratory.

Database organization and coding details. A third problem is the comprehensibility and interpretability of the structure and organization of the data set, especially the relationship among the questions within a data set. For example, the $N L S Y$ code for "Valid Skip" (-4) might be given under any of the following circumstances: a male R ("Respondent") when asked a question about date of most recent abortion; a female $\mathrm{R}$ in 1989 when asked a question about the sex of a first child when the same question was answered in 1987; any $\mathrm{R}$ when asked a question about date of birth if the same question was answered in the previous year; and so forth. 
That a longitudinal database may extend across many years is often itself a cause for confusion. For example, many questions are repeated, sometimes at irregular intervals, over the years; students commonly select questions from different years, which can create problems in interpretation if a cross-sectional study was intended.

Sometimes the wording of questions is obscure, for example, "R27412. [Q16.186] INT CHECK - IS THERE A 7TH AGE OR PARENT TYPE? - TIME 6" is baffling in isolation. In the $N L S Y$, every variable is identified by a variable name, called an "R-number," and a question name. The question name identifies the variable's place in the questionnaire, but the form of the number is not consistent for all years. "INT CHECK" (interviewer check) questions are questions that are answered by the interviewer, not the respondent; this might be done to check, for example, a previous answer, in order to skip to a different question (S. McClaskie, personal communication, February 26, 2001).

Errors. Large data sets not uncommonly contain errors of various sorts. In some cases, these are simply wrongly coded or transcribed data; these errors, when discovered, may be announced and corrected by the producer of the data archive. Bulletins in which errors are reported to users may be issued. In other cases, data may be erroneously reported by the respondent. For example, a sizeable number of young respondents reported spending more than $24 \mathrm{~h}$ per day watching TV in the NLSY database. Pointing these out provides an opportunity for the teacher to engage students in a discussion of the ambiguities of data and what to do about them.

\section{Using a CD-ROM Database: \\ A Run-Time Example}

In this section, the NLSY (Center for Human Resources Research, 1998) will be used to illustrate the selection of variables and the extraction of data. Other databases have different programs and methods, but many share the common elements shown here.

As an example, I will suppose the student user has identified the topic area as the relationship among age of first sexual intercourse, drug use, and self-esteem. In my classes, I provide a list of some $450+$ descriptors that appear in the database prior to opening the database for student access; this list gives the student an idea of the wide variety of data that are available and encourages him or her to think creatively about a topic to explore. Having selected a topic, the student begins the interactive process of narrowing his or her focus by searching for information within the database that will yield answers to questions for which answers are sought. The database search engine, CHRR NLS Database Investigator (Center for Human Resources Research, 1999), called DB' Gator, is used to search the database, as is shown in Figure 1.

First, a data set cohort is identified. Here, the original 1979 cohort is selected, as is shown at pointer 1 in the figure. Next, the method of searching the question items in the database is specified; for this example, I chose to search by "Any word in context," as is shown at pointer 2 . At pointer 3, the search term is entered into the search window, and the search engine locates matches to words within which the search term occurs, as is shown at pointer 4. At pointer 5, DB' Gator indicates the number of question items that contain the word searched. By dragging multiple words from the window above the Result window, additional terms can be added as Boolean specifications. When these search criteria are applied, the matching questions are presented in the Variables List window, along with a check box that enables the user to tag variables as ones to be extracted (pointer 6). The codebook description of a variable (example shown at pointer 7) can be obtained with a mouse click and includes such information as the exact wording of the question, the code values and the frequency of each response, as well as the previous and following question asked of the respondents (useful for understanding certain missing code values or related questions).

After variable selection, the data extraction specifications are entered. Figure 2 shows the Data Extraction window (pointer 1) for 18 variables selected for this example. The user may select to extract a limited number of cases, in order to reduce time or space demands (pointer 2), a sequential subset of cases (pointer 3), or a selected number of cases from a sequential subset. Four data file formats are available (pointer 4), and control statements may be produced for SAS and SPSS, along with the codebook information (pointer 5). The three files produced include the data file, the codebook file, a .doc file listing the variable name, the question, the number of cases, the mean, the maximum, the minimum, and the column numbers and length for each variable (pointer 6). The entire extraction process is very rapid; in the present instance (selecting only 1 of 50 cases), it took only $11 \mathrm{sec}$ (pointer 7).

Although few databases provide tools as sophisticated and user friendly as the $N L S Y$ DB' Gator, several include search and extraction tools that incorporate important functions provided by DB' Gator. The National Opinion Research Center's GSS Data and Information Retrieval System (1999) is a set of programs that are comparable to DB' Gator in nearly every respect and also enable online cross-tabulation and multiple regression.

\section{Student Responses}

The National Longitudinal Surveys (NLSs) are designed for use by knowledgeable professionals. Undergraduate students face a steep learning curve in order to master their use. For students with little background in using computers, even DB' Gator is not readily accessible.

Despite these potential sources of confusion, students generally responded favorably to the $N L S Y$ project. A question on my end of term student course evaluation asks students to rate on a 5-point scale the "usefulness" of the three projects required for the course. The mean rating of the $N L S Y$ project generally falls between that 


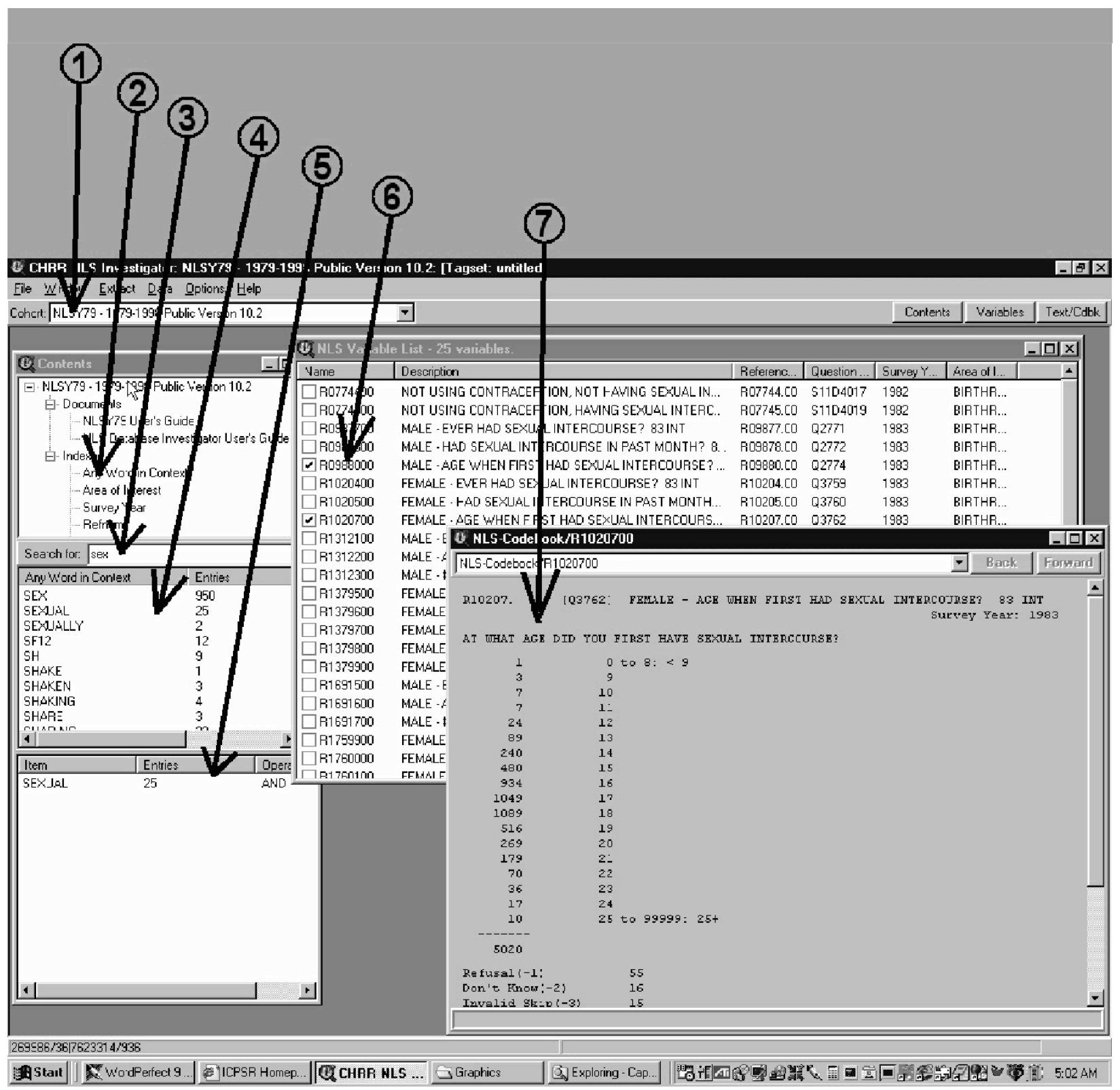

Figure 1. CHRR NLS Database Investigator (DB'Gator) screen showing selection of two variables from the National Longitudinal Survey of Youth database. Codebook information for the second variable is shown in the lower right window.

for the observational study, usually judged least useful, and that for the individually designed experimental project, usually judged most useful.

\section{Some Data Archives Suitable for Use in Teaching Undergraduate Research Methods}

Below are listed some databases that can be readily obtained on line or in CD-ROM format and that may be suitable for student use. I have not examined all of these archives in detail, but I have verified that they exist and can be either directly accessed or obtained on a CDROM. I have excluded databases such as High School and Beyond (National Center for Education Statistics, 2000), which restrict access to faculty or bonafide researchers. Nearly all of these, and many more, are linked from the Inter-university Consortium for Political and Social Research (ICPSR) at http://www.icpsr.umich.edu.

General Social Survey (GSS) at National Opinion Research Survey (NORC). The GSS has been conducted by NORC annually since 1972 (except for 3 years). For each round of surveys, the Roper Center for Public Opinion Research prepares a cumulative data set that merges previous years of the GSS into a single file, with each year or survey constituting a subfile. Main areas covered in 


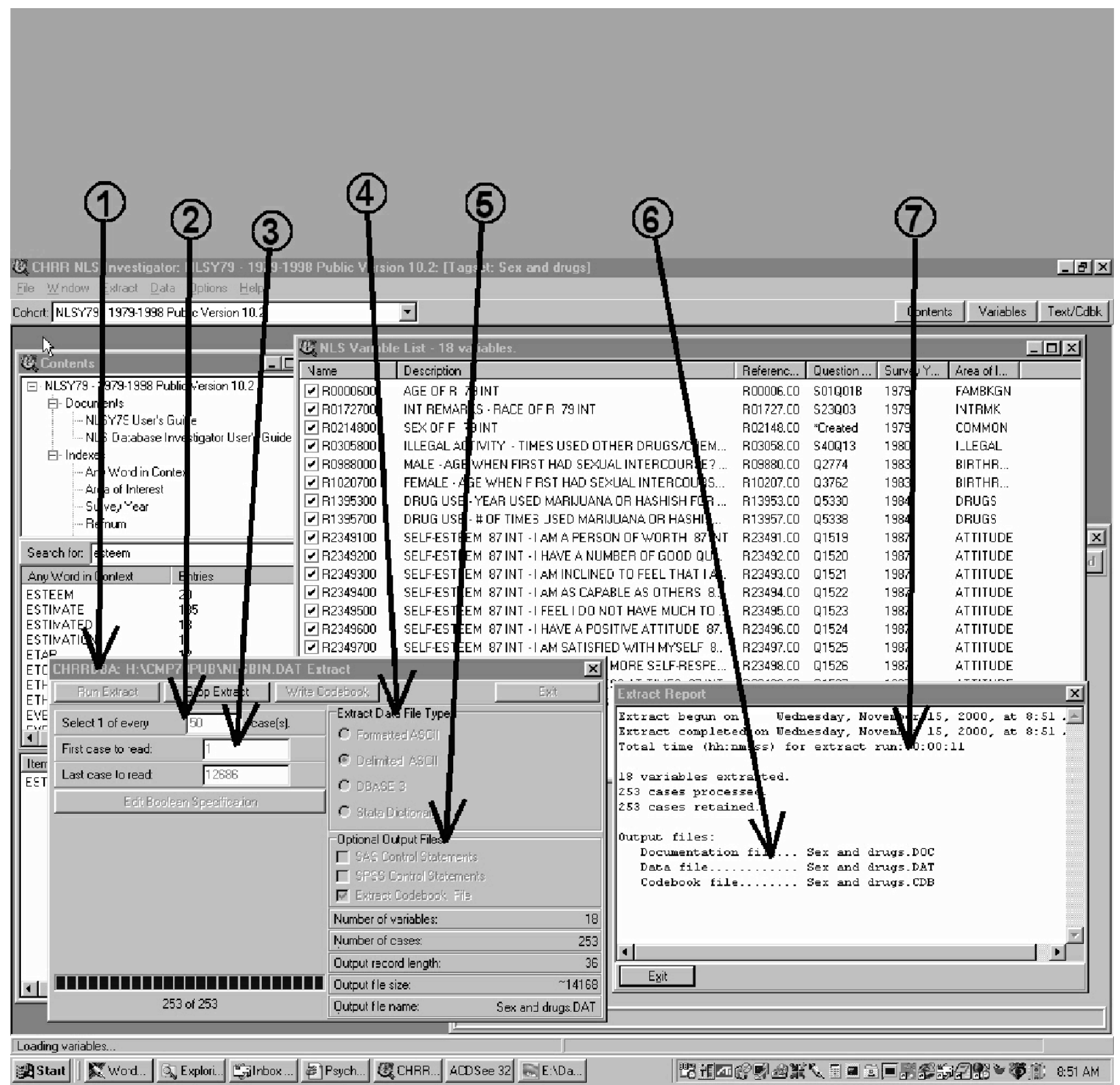

Figure 2. CHRR NLS Database Investigator (DB'Gator) screen showing results of extraction of 18 variables. Only 1 of 50 , or 253 cases, have been extracted; the data are saved as a delimited ASCII file, and the codebook file is written to disk.

the GSS include socioeconomic status, social mobility, social control, the family, race relations, sex relations, civil liberties, and morality. Topical modules containing a selected subset of related variables designed to investigate new issues or to expand the coverage of an existing subject have been part of the GSS since 1977, when the first module on race, abortion, and feminism appeared. This site allows on-line selection of variables for downloading and cross-tab calculations on line similar to (although with fewer options than) DB' Gator and provides a list of references for all variables. This is a huge but eas- ily accessible collection; access via the WWW enhances the usability of this database.

National Longitudinal Surveys. The National Longitudinal Survey of Youth (NLSY79) is a multipurpose survey that originally included more than 12,600 individuals in the U.S. who have been interviewed since 1979, when they were 14-21 years of age. It contains extensive information about the employment, education, training, and family experiences of the respondents. Since 1982, questions have been asked about pregnancy, postnatal fertility, and child care experiences of the female respon- 
dents in the NLSY79 sample. Since 1986, assessments have been administered to the children of NLSY79 mothers, to measure their cognitive ability, temperament, motor and social development, behavior problems, perceived competence, and the quality of their home environment. Data from older children focus on education, employment, and family-related behaviors and attitudes; a selfadministered paper questionnaire asks about a variety of more personal attitudes and behaviors, including questions relating to substance use, early sexual activity, and other nonnormative activities. The NLSY79 Young Adult data collection permits researchers to examine associations between a young adult's family background, intellectual and emotional development, and incipient career development activities. The Windows-based CHRR NLS Database Investigator (DB'Gator; Center for Human Resources Research, 1999) data extraction program is demonstrated above.

Additional data sets include Mother-Child Data (CDROM; see Sheets, 1991, for further information), Old Cohorts (CD-ROM; for men 45-59 years of age in 1966, men 14-24 in 1966, women 30-44 in 1967, and women 14-24 in 1968), and the Original Cohorts (for mature women 30-44 in 1967, young women 14-24 in 1968, and mature women in the year 1989).

Ordering information for the databases, which are available only on CD-ROM, can be found at http://www. nlsbibliography.org/display_db.php3. Most of the NLS CDs cost \$20; the DB' Gator search program can be downloaded at no cost.

National Survey of Families and Households. Archives include studies of family life, careers, psychological development, mental health and other topics (Center for Demography and Ecology, University of Wisconsin, Madison, 4412 Social Science Building, 1130 Observatory Drive, Madison, WI 53706-1393).

PsychExps. Based on the projects created by students and faculty using this data collection system, this site includes many well-designed experiments for student projects; it enables downloading of data collected from experiments. See Williams et al. (1999) for further information on this site (http://www.olemiss.edu/psychexps/).

UK Data Archive. A collection of accessible computerreadable data in the social sciences and humanities. I have no experience with this site (http://www.data-archive. ac.uk/).

U.S. Census data. Census reports and links to other federal government and international agencies offering statistical reports. My students have found this site complicated and difficult to use (http://www.census.gov).

Wisconsin Longitudinal Study. A 35-year study of a random sample of 10,317 men and women who graduated from Wisconsin high schools in 1957. Survey data collected provide a full record of social background, youthful aspirations, schooling, military service, family formation, labor market experiences, and social participation. Survey data have been supplemented by mental ability tests (of primary respondents and 2,000 of their siblings), measures of school performance, and characteristics of communities of residence, schools and colleges, employers, and industries. I have not accessed data here (Data and Program Library Service, University of Wisconsin, Madison).

\section{URLs That Link to Archives and/or Include Information on Database Availability}

Data on the Net is a listing of 851 Internet sites of numeric social science statistical data, data catalogs, data libraries, social science gateways, addresses, and more (at the University of California, San Diego; http://odwin.ucsd. edu/idata/).

The Inter-university Consortium for Political and Social Research (ICPSR) is part of the Institute for Social Research at the University of Michigan. This site contains a great many links to databases, including most of those mentioned above. It is believably said to access the world's largest archive of computerized social science data and is probably the best starting place for a search. Click "topical archives" (http://www.icpsr.umich.edu/topical.html) for categories within which many other data sets may be accessed.

The Gallup Organization. This is a provider of public opinion poll data. It provides mostly summary data, rather than raw data, but often is useful for examples (http://www. gallup.com).

The Harris Poll. This site contains the latest Harris poll and comparisons of the previous poll's telephone responses with Internet responses. It provides mostly summary data, rather than raw data (http://techsetter.com/ harris/html/home.html).

\section{Other Sources of Information \\ on Archives and Databases}

Inter-University Consortium for Political and Social Research, University of Michigan, Institute for Social Research, P. O. Box 1248, Ann Arbor, MI 48106.

University of Connecticut Institute for Social Inquiry, Roper Center for Public Opinion Research, Users Services Development, 341 Mansfield Road, Room 421, Box U-164, Storrs, CT 06268.

Zill, N., \& Daly, M. (1993). Researching the family: A guide to survey and statistical data on U.S. families. Washington, DC: US Department of Health and Human Services.

\section{REFERENCES}

Behavior Science Database Consortium (1999). Characteristics of psychological research and implications for a psychology database [On-line]. Available: http://www.am.org/federation/database.html. [Retrieved June 6, 2000.]

Bordens, K. S., \& Аввотт, B. B. (1996). Research design and methods: A process approach (3rd ed.). Mountain View, CA: Mayfield.

Center for Human Resources Research (1998). National longitudinal survey of youth. Columbus, $\mathrm{OH}$ : Author.

Center for Human Resources Research (1999). CHRR NLS Database Investigator [computer program]. Columbus, $\mathrm{OH}$, Author. 
Davis, J. A., \& Sмiтh, T. W. (1997). General social survey, 1972-1996 cumulative file [Computer file]. Chicago: National Opinion Research Center [producer]. Ann Arbor, MI: Interuniversity Consortium for Political and Social Research [distributor].

Drake, M. A., \& Stahl, J. M. (1995). Design and use of a U.S. census data computer laboratory for teaching undergraduate research methods. Behavior Research Methods, Instruments, \& Computers, 27, 285-288.

Elmes, D. G. Kantowitz, B. H., \& Roediger, H. L., III (1999). Research methods in psychology (6th ed). Pacific Grove, CA: Brooks/ Cole.

HeIman, G. (1995). Research methods in psychology. Boston: Houghton Mifflin.

Hewson, C. M., Laurent, D., \& Vogel, C. M. (1996). Proper methodologies for psychological and sociological studies conducted via the Internet. Behavior Research Methods, Instruments, \& Computers, 28, 186-191.

Liebert, R., \& Liebert, L. (1995). An introduction to methods of psychological research (4th ed.). Englewood Cliffs, NJ: Prentice-Hall.

McCall, R, \& Appelbaum, M. (1991). Some issues of conducting secondary analyses. Developmental Psychology, 27, 911-918.

National Center for Education Statistics (2000). High school and beyond. Washington, DC: Author.

National Opinion Research Center (1999). GSS data and information retrieval system [Computer program]. Chicago: University of Chicago.
Oehlmann, R. (1998, November). Publishing psychological experiments: A data model to support acquisition and distribution. Paper presented at the 1998 Society for Computers in Psychology conference, Dallas. Abstract retrieved November 15, 2000 from http://ctiwebct. york.ac.uk/LTSNCiPAbstracts/cip98.htm.

Pachnowski, L., Newman, I., \& JuRCZYK, J. (1997). Immediate data: The World Wide Web as a resource for teaching research methods [Online]. Available: http://junior.apk.net/ jurczyk/eera.html. [Retrieved November, 2000.]

RAY, W. (2000). Methods toward a science of behavior and experience (6th ed.). Belmont, CA: Wadsworth/Thomson Learning.

SheEts, C. T. (1991). NLSDBA: The National Longitudinal surveys on compact disk. Behavior Research Methods, Instruments, \& Computers, 23, 212-213.

Singleton, R, Straits, B., \& Straits, M. (1993). Approaches to social research (2nd ed.). New York: Oxford University Press.

VAdUm, A., \& RANkin, N. (1998). Psychological research: Methods of discovery and validation. New York: McGraw-Hill.

Williams, J. E., McGraw, K. O., \& Tew, M. D. (1999). Undergraduate labs and computers: The case for PsychExps. Behavior Research Methods, Instruments, \& Computers, 31, 287-291.

(Manuscript received November 24, 2000; revision accepted for publication February 10, 2001.) 